\title{
Triglyceride Up-regulates Expression of ABCG1 in PMA-induced THP-1 Macrophages Through Activation of JNK and p38 MAPK Pathways
}

\author{
Jaewon Lim ${ }^{1}$, Sung Hoon Kim ${ }^{1}$, Yeo Wool Kang ${ }^{1}$, Byung Chul Jung ${ }^{1}$, Hyun-Kyung Kim ${ }^{2}$, \\ Juyeon Lee ${ }^{1}$, Dongsup Lee ${ }^{3}$, Ki-Jong Rhee ${ }^{1, \uparrow}$ and Yoon Suk Kim ${ }^{1, \uparrow}$ \\ ${ }^{1}$ Department of Biomedical Laboratory Science, College of Health Sciences, Yonsei University, \\ Wonju, Gangwon-do 220-710, Korea \\ ${ }^{2}$ Department of Biomedical Laboratory Science, College of Natural Science, Gimcheon University, \\ Gimcheon 740-704, Korea \\ ${ }^{3}$ Department of Clinical Laboratory Science, Hyejeon College, Hongseong, \\ Chungcheongnam-do 350-702, Korea
}

\begin{abstract}
Triglyceride (TG) can cause death of macrophages and formation of foam cells thereby increasing inflammation in atherosclerotic plaques. Accumulation of cholesterol in macrophages is another critical event that promotes development of inflammatory cardiovascular diseases. Several proteins are known to transport intracellular cholesterol outside of the cell and these proteins are thought to be protective against atherosclerosis pathogenesis. It is unknown whether TG can affect cholesterol efflux in macrophages. In the current study, we examined mRNA expression levels of genes that promote efflux of cholesterol (ABCA1, ABCG1 and SR-B1). We found that TG treated THP-1 macrophages exhibited an increase in ABCG1 expression in a dose- and time-dependent manner. In contrast, the expression of ABCA1 and SR-B1 remained unchanged. To identify cell signaling pathways that participate in up-regulation of ABCG1, THP-1 macrophages were treated with various cell signaling inhibitors. We found that inhibition of the JNK and p38 MAPK pathway completely abrogated up-regulation of ABCG1 whereas inhibition of MEK1 further enhanced ABCG1 expression in TG treated THP-1 macrophages. Also, TG induced phosphorylation of JNK and p38 MAPK in THP-1 macrophages. These results suggest that TG may potentially influence cholesterol efflux in macrophages.
\end{abstract}

Key Words: THP-1 macrophages, Triglyceride (TG), ABCG1, JNK, p38 MAPK

\section{INTRODUCTION}

Triglyceride (TG) is one of the main causes of cardiovascular disease especially atherosclerosis. TG can damage

\footnotetext{
*Received: December 1, 2014 / Revised: December 22, 2014

Accepted: December 22, 2014

${ }^{\dagger}$ Corresponding author: Yoon Suk Kim. Department of Biomedical Laboratory Science, College of Health Science, Yonsei University, Wonju 220-710, Korea.

Tel: +82-33-760-2860, e-mail: yoonsukkim@yonsei.ac.kr

${ }^{\dagger}$ Corresponding author: Ki-Jong Rhee. Department of Biomedical Laboratory Science, College of Health Science, Yonsei University, Wonju 220-710, Korea.

Tel: +82-33-760-2445, e-mail: kjrhee@yonsei.ac.kr

(C) The Korean Society for Biomedical Laboratory Sciences. All rights reserved.
}

vascular epithelium and cause inflammatory response in immune cells eventually leading to atherosclerosis (Lind, 2003; Aronis et al., 2005; Kastelein et al., 2008). Several studies have shown that addition of exogenous TG results in intracellular accumulation of TG, leading to multiple inflammatory effects in macrophages, such as cell death by nitric oxide (NO) formation and expression of $\mathrm{CD}$ markers not expressed in resting macrophages (Aronis et al., 2005; Feingold et al., 2012; Son et al., 2013). In early stages of atherosclerosis, accumulation of cholesterol in macrophages is regarded as another important event that precedes subsequent development of atherosclerosis. Recent studies indicate that cholesterol efflux is thought to be a major 
process involved in plaque formation in atherosclerotic regions (Tall et al., 2008; Moore and Tabas, 2011). Cholesterol homeostasis is a balance between intake/efflux of cholesterol in cells (Daniels et al., 2009). In an epidemiological study of atherosclerosis patients, cholesterol efflux was a strong inverse predictor of disease (Khera et al., 2011). Palmer et al., reported that TG-rich lipoproteins inhibit cholesterol efflux from human macrophage foam cell to promote formation of atherosclerotic lesion (Palmer et al., 2004).

Scavenger receptor class B1 (SR-B1) is a high density lipoprotein (HDL) receptor that induces cholesterol efflux in macrophages and thereby protecting against the development of atherosclerosis (Wang et al., 2001; Kunjathoor et al., 2002; Yancey et al., 2003; Kennedy et al., 2005; Moore and Tabas, 2011). Another class of proteins called ATPbinding cassette $(\mathrm{ABC})$ transporters moves cholesterol outside of the cell and regulates cellular lipid homeostasis in macrophages. The ABC transporter proteins bind ATP and use the energy to drive the translocation of a diverse array of compounds across specific cellular membrane bilayers that include the endoplasmic reticulum, mitochondria, peroxisomes and plasma membrane (Dean et al., 2001; Tarr and Edwards, 2008). ABCA1 and ABCG1 are members of $\mathrm{ABC}$ transporter superfamily and mediate cholesterol efflux in macrophages (Moore and Tabas, 2011). In the current study, we determined whether TG induced changes in expression of genes involved in cholesterol efflux. We found that exogenous TG treated THP-1 macrophages up-regulated expression of ABCG1. Up-regulation of ABCG1 occurred through activation of the JNK and p38 MAPK pathways. Our results suggest that TG may modulate cholesterol homeostasis in THP-1 macrophages.

\section{MATERIALS AND METHODS}

\section{Materials}

Phorbol 12-myristate 13-acetate (PMA) was purchased from Sigma-Aldrich (St. Louis, MO, USA). TG emulsion (Lipofundin ${ }^{\circledR}$ MCT/LCT 20\%) was purchased from B. Braun Melsungen AG (Melsungen, Germany). Lipofundin ${ }^{\circledR}$ MCT/LCT 20\% was used to deliver TG into cells as previously described (Aronis et al., 2005). Trypsin-EDTA was obtained from Gibco BRL (Grand Island, NY, USA). TRIzol $^{\circledR}$ for RNA isolation was obtained from Invitrogen (Carlsbad, CA, USA). Chemical inhibitors for classical PKC (RO31-7549, 0.5 $\mu \mathrm{M}$ ), MEK1 (PD98059, $50 \mu \mathrm{M}$ ), PI3K (LY294002, $10 \mu \mathrm{M})$, p38 MAPK (SB203580, $20 \mu \mathrm{M}$ ), JNK (SP600125, $50 \mu \mathrm{M})$ and cRAF-1 kinase (GW5074, $0.5 \mu \mathrm{M}$ ) were purchased from Calbiochem (Darmstadt, Germany). Specific inhibitor of NF-кB (BAY11-7085, 1 $\mu \mathrm{M}$ ) was purchased from Enzo Life Sciences (New York, NY, USA). Specific inhibitor of PLC (U73122, $50 \mu \mathrm{M}$ ) was purchased from Cayman Chemical (Ann Arbor, MI, USA). Dimethyl sulfoxide (DMSO) was obtained from Sigma-Aldrich (St. Louis, MO, USA). All inhibitors were dissolved as stock solutions in DMSO and stored at $-20^{\circ} \mathrm{C}$ prior to usage. Antibodies specific for phosphorylated JNK (p-JNK) and phosphorylated p-38 MAPK (p-p38 MAPK) were purchased from Cell Signaling Technology (Danvers, MA, USA). Antibody specific for $\beta$-actin was purchased from Santa Cruz Biotechnology (Santa Cruz, CA, USA).

\section{Cell culture}

The THP-1 human acute monocytic leukemia cell line (ATCC, Manassas, VA, USA) was grown in RPMI 1640 media supplemented with $10 \%$ fetal bovine serum (FBS) and penicillin-streptomycin and maintained at $37^{\circ} \mathrm{C}$ in a humidified atmosphere with $5 \% \mathrm{CO}_{2}$. To induce differentiation of THP-1 cells into macrophages, cells were seeded in 6-well plates at a density of $1 \times 10^{6}$ cells/well and treated with PMA (200 nM) for $48 \mathrm{~h}$. Thereafter, PMAdifferentiated THP-1 macrophages were incubated with TG and/or chemical inhibitors.

\section{RNA extraction and semi-quantitative reverse trans- criptase PCR (RT-PCR)}

Total RNA was isolated from THP-1 macrophages using TRIzol $^{\circledR}$ reagent according to the manufacturer's instructions. cDNA was synthesized by reverse transcription with $2 \mu \mathrm{g}$ total RNA, $0.25 \mu \mathrm{g}$ of random hexamer (Invitrogen) and 200 unit of Moloney murine leukemia virus reverse transcriptase (MMLV-RT; Invitrogen) for $10 \mathrm{~min}$ at $25^{\circ} \mathrm{C}, 50 \mathrm{~min}$ at $37^{\circ} \mathrm{C}$ and $15 \mathrm{~min}$ at $70^{\circ} \mathrm{C}$. cDNA was PCR amplified 
using Prime Taq premix PCR kit (Genet Bio, Chungnam, Korea) for 25 50 cycles using specific primers. Primer sequences are as follows : ABCA1; 5'-CCC CTG TTT CCG TTA CCC-3' (forward), 5'-AGC CCT CAG CAT CTT GTC-3' (reverse), ABCG1; 5'-GCA GTT ACT CTG CAG AGA TG-3' (forward), 5'-CGG AAA TTC CTT TCA GGA GG-3' (reverse), SR-B1; 5'-CTG TGG GTG AGA TCA TGT GG-3' (forward), 5'-GCC AGA AGT CAA CCT TGC TC-3' (reverse). GAPDH was used as an internal control (Yoon et al., 2012). PCR products were electrophoresed on $2 \%(\mathrm{w} / \mathrm{v})$ agarose gels containing $0.5 \mu \mathrm{g} / \mathrm{mL}$ ethidium bromide and the product size determined by comparison to 100 bp DNA ladder marker (Intron, Gyeonggi, Korea). Gel images were taken using Gel Doc ${ }^{\mathrm{TM}} \mathrm{XR}+$ system (Bio-Rad, Hercules, CA, USA). The PCR product band intensity was measured and normalized against GAPDH using Image $\mathrm{Lab}^{\mathrm{TM}}$ software (version 4.1, Bio-Rad).

\section{Western-blot analysis}

Cells were washed with PBS and then lysed at $4^{\circ} \mathrm{C}$ with a lysis buffer containing 1\% Triton X-100, protease inhibitor cocktail (Sigma-Aldrich, St. Louis, MO, USA), phosphatase inhibitor cocktail (Roche, Basel, Switzerland) and PBS. The lysate was centrifuged at 14,000 RPM for $5 \mathrm{~min}$ at $4{ }^{\circ} \mathrm{C}$. The supernatant was collected and proteins were quantified using Lowry protein assay. Protein samples (60 $\mu \mathrm{g}$ per lane, but $20 \mu \mathrm{g}$ per lane in $\beta$-actin analysis) were separated by $12 \%$ sodium dodecyl sulfate (SDS)-polyacrylamide gel electrophoresis and transferred to nitrocellulose membrane. The membrane was blocked with TBST containing 5\% (w/v) skim milk for $30 \mathrm{~min}$ and then incubated with primary antibody overnight at $4^{\circ} \mathrm{C}$. Each immunoblot was then incubated with appropriate horseradish peroxidaselabeled secondary antibody (anti-mouse or anti-rabbit). The antibody-labeled proteins were visualized using ECL kit (Thermo, Waltham, MA, USA) and exposed onto X-ray film (AGPA, Mortsel, Belgium). $\beta$-actin was used as an internal control.

\section{Statistical analysis}

$P$-values were calculated using the Student's $t$-test. Values are shown as mean and standard error of the mean
(SEM). Each experiment was conducted three times and the data pooled for analysis.

\section{RESULTS}

\section{ABCG1 expression is up-regulated in TG treated THP-1 macrophage}

Cholesterol accumulation in macrophages promotes atherosclerosis and several proteins are known to regulate cholesterol movement in cells. In this current study, we first assessed whether TG could affect the expression of cholesterol regulatory genes in THP-1 macrophages. PMAinduced THP-1 macrophages were treated with TG (1 mg/

A TG (1 mg/ml)

B

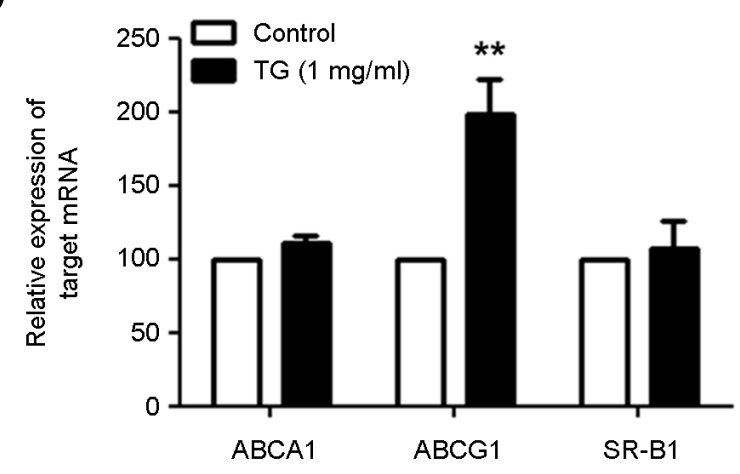

Fig. 1. TG treated THP-1 macrophages up-regulates ABCG1 mRNA expression. THP-1 macrophages were incubated with TG $(1 \mathrm{mg} / \mathrm{mL})$ for $24 \mathrm{~h}$ and RT-PCR analysis were performed on ABCA1, ABCG1 and SR-B1. The PCR products were electrophoresed on a $2 \%$ agarose gel. (A) A representative image of ABCA1, ABCG1 and SR-B1 expression. (B) Densitometry analysis of PCR products. ABCA1, ABCG1 and SR-B1 expression level were normalized to GAPDH expression. THP-1 macrophages without TG treatment $(0 \mathrm{mg} / \mathrm{mL})$ were set as 100 . Data are expressed as the mean \pm SEM from three independent experiments. $P$-values were determined by the Student's $t$-test $(* * P<0.01)$. 
A

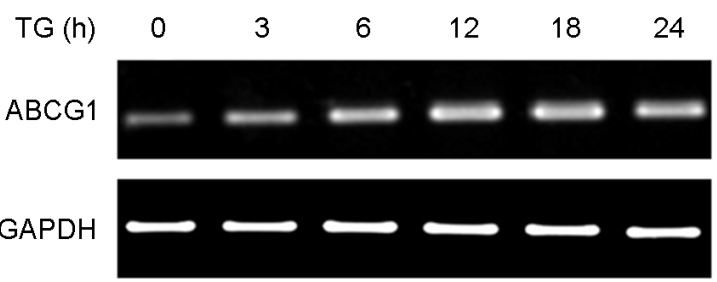

B

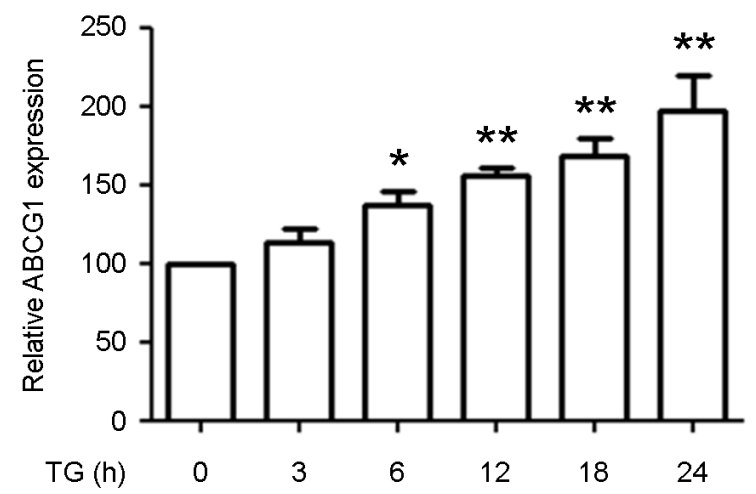

Fig. 2. TG treated THP-1 macrophages up-regulate ABCG1 expression in a time-dependent manner. THP-1 macrophages were incubated with TG $(1 \mathrm{mg} / \mathrm{mL})$ for the indicated times $(0,3$, $6,12,18$, and $24 \mathrm{~h}$ ) and ABCG1 expression assessed by RT-PCR. The PCR products were electrophoresed on a $2 \%$ agarose gel. (A) A representative image of ABCG1 expression. (B) Densitometry analysis of PCR products. The ABCG1 expression level was normalized to GAPDH expression. Time point $0 \mathrm{~h}$ was set as 100 . Data are expressed as the mean \pm SEM from three independent experiments. $P$-values were determined by the Student's $t$-test $\left(* P<0.05,{ }^{*} * P<0.01\right)$.

$\mathrm{mL}$ ) for $24 \mathrm{~h}$ and expression of ABCA1, ABCG1 and SR-B1 assessed by RT-PCR. We found that expression of ABCG1 was increased by TG treatment in THP-1 macrophages whereas expression of ABCA1 and SR-B1 remained unchanged (Fig. 1). These results indicate that TG treatment up-regulated mRNA expression of ABCG1 in THP-1 macrophages suggesting that TG can potentially enhance cholesterol efflux in macrophages.

\section{TG up-regulates ABCG1 expression in THP-1 macro- phages in a time- and dose-dependent manner}

Since TG up-regulated ABCG1 expression in THP-1 macrophages at $24 \mathrm{~h}$, we next determined when TG induced expression of ABCG1. THP-1 macrophages were treated
A

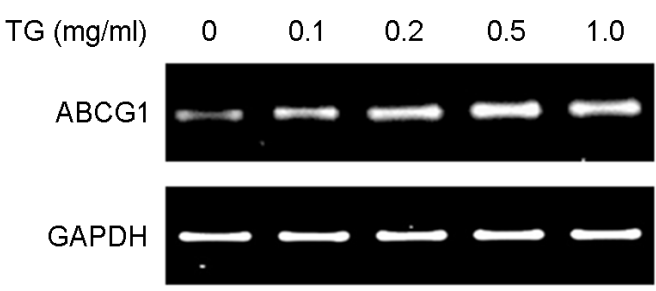

B

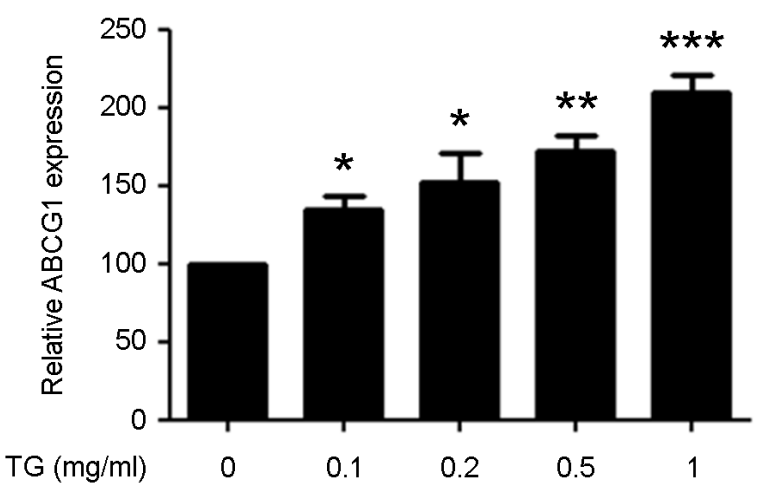

Fig. 3. TG up-regulates ABCG1 expression in THP-1 macrophages in a dose-dependent manner. THP- 1 macrophages were incubated with indicated concentrations of $\mathrm{TG}(0,0.1,0.2,0.5$, and $1.0 \mathrm{mg} / \mathrm{mL}$ ) for $24 \mathrm{~h}$ and ABCG1 expression assessed by RT-PCR. The PCR products were electrophoresed on a $2 \%$ agarose gel. (A) A representative image of ABCG1 expression. (B) Densitometry analysis of PCR products. The ABCG1 expression level was normalized to GAPDH expression. THP-1 macrophages without TG treatment $(0 \mathrm{mg} / \mathrm{mL})$ were set as 100 . Data are expressed as the mean \pm SEM from three independent experiments. $P$-values were determined by the Student's $t$-test $\left({ }^{*} P<0.05,{ }^{* *} P<0.01,{ }^{* * *} P<\right.$ $0.001)$.

with TG $(1 \mathrm{mg} / \mathrm{ml})$ for the indicated times $(0,3,6,12,18$ and $24 \mathrm{~h}$ ) and ABCG1 expression assessed by RT-PCR analysis. We found that ABCG1 expression began to increase by $6 \mathrm{~h}$ after TG treatment and continued to increase for $24 \mathrm{~h}$ (Fig. 2). We next determined the concentration at which TG could up-regulate ABCG1 expression. THP-1 macrophages were treated with TG $(0,0.1,0.2,0.5,1.0$ $\mathrm{mg} / \mathrm{mL}$ ) for $24 \mathrm{~h}$ and ABCG1 expression assessed by RT-PCR. We found that $0.1 \mathrm{mg} / \mathrm{mL}$ of TG was sufficient to induce ABCG1 expression by $24 \mathrm{~h}$ (Fig. 3). These results indicate that gene expression of ABCG1 in THP-1 macrophages is up-regulated in a time- and dose-dependent manner by TG treatment. 
A

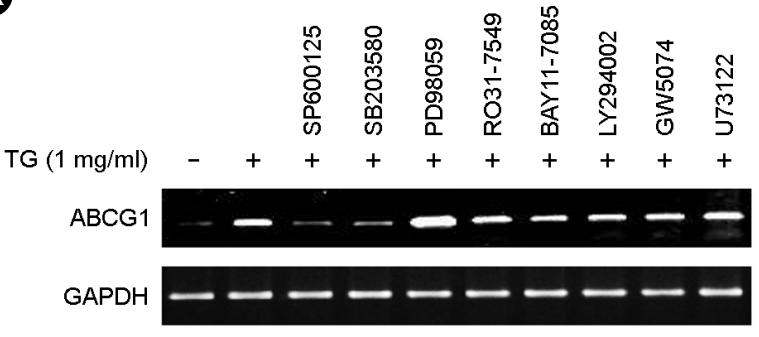

B

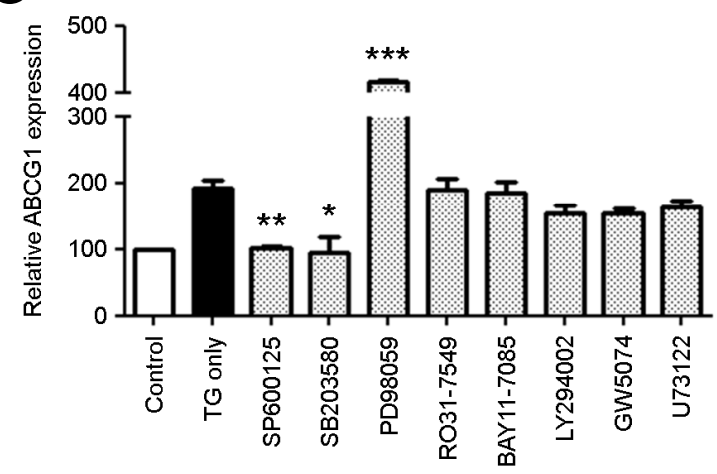

C

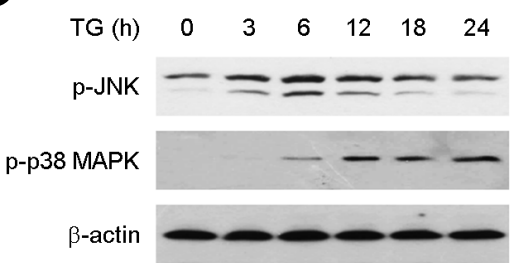

Fig. 4. Modulation of ABCG1 expression in TG treated THP-1 macrophages is mediated by JNK, p38 MAPK and MEK1 pathways. TG $(1 \mathrm{mg} / \mathrm{mL})$ treated THP-1 macrophages were cocultured with chemical inhibitors for $24 \mathrm{~h}$ and ABCG1 expression assessed by RT-PCR. The inhibitors used are as follows: RO317549 (PKC inhibitor), BAY11-7085 (NF-kB inhibitor), LY294002 (PI3K inhibitor), GW5074 (cRAF-1 kinase inhibitor), U73122 (PLC inhibitor), SP600125 (JNK inhibitor), PD98059 (MEK1 inhibitor) and SB203580 (p38 MAPK inhibitor). The PCR products were electrophoresed on a $2 \%$ agarose gel. (A) A representative image of ABCG1 expression. (B) Densitometry analysis of PCR products. The ABCG1 expression level was normalized to GAPDH expression. THP-1 macrophages without TG treatment $(0 \mathrm{mg} / \mathrm{mL})$ were set as 100 . Data are expressed as the mean \pm SEM from three independent experiments. $P$-values were determined by the Student's $t$-test $(* P<0.05, * * P<0.01, * * * P<0.001)$. (C) THP-1 macrophages were incubated with TG $(1 \mathrm{mg} / \mathrm{mL})$ for the indicated times $(0,3,6,12,18$, and $24 \mathrm{~h})$ and cell extracts were separated on $12 \%$ SDS-polyacrylamide gel and transferred to nitrocellulose membrane. Phosphorylated-JNK (p-JNK) and phosphorylated-p38 MAPK (p-p38 MAPK) were detected by Western-blot using antip-JNK antibody and anti-p-p38 MAPK antibody. $\beta$-actin was used as an internal control. A representative image is shown from three independent experiments.
Modulation of ABCG1 expression in TG treated THP-1 macrophages is mediated by JNK, p38 MAPK and MEK1 pathways

To identify cell signaling pathways involved in upregulation of ABCG1 in TG treated THP-1 macrophages, TG treated THP-1 macrophages were co-treated with chemical inhibitors of select cell signaling pathways and ABCG1 expression assessed by RT-PCR analysis. The following inhibitors were tested: PKC (RO31-7549), MEK1 (PD98059), NF-kB (BAY11-7085), PI3K (LY294002), JNK (SP600125), p38 MAPK (SB203580), cRAF-1 kinase (GW5074) and PLC (U73122). We found that the JNK inhibitor (SP600125) and p38 MAPK inhibitor (SB203580) dramatically blocked ABCG1 up-regulation (Fig. 4A and Fig. 4B). In contrast, the MEK1 inhibitor (PD98059) further enhanced ABCG1 expression. To confirm activation of the JNK and p38 MAPK in TG treated THP-1 macrophages, THP-1 macrophages were treated with TG $(1 \mathrm{mg} / \mathrm{mL})$ for the indicated times $(0,3,6,12,18$ and $24 \mathrm{~h})$ and protein levels of p-JNK and p-p38 MAPK were detected by Western-blot analysis. We found that $\mathrm{p}-\mathrm{JNK}$ and $\mathrm{p}-\mathrm{p} 38$ MAPK were also increased in TG time-dependent manner (Fig. 4C). In particular, TG transiently activated JNK at $3 \mathrm{~h}$. However, p38 MAPK was activated by TG at $6 \mathrm{~h}$ and remained activated for an additional $24 \mathrm{~h}$. These results suggest that TG-induced up-regulation of ABCG1 expression requires activation of the JNK and p38 MAPK. Furthermore, MEK1 negatively regulates ABCG1 gene expression in TG treated THP-1 macrophages.

\section{DISCUSSION}

Uptake of TG and cholesterol by macrophages both contribute to development atherosclerosis. As far as we know, the results from this current study are the first to demonstrate that uptake of TG by macrophages can modulate expression of genes involved in cholesterol efflux. The key finding of our study is that TG can induce expression of the cholesterol efflux gene, ABCG1, via activation of the JNK and p38 MAPK pathways. In addition, we identified the MEK1 pathway as a negative regulator of 
TG induced ABCG1 up-regulation in THP-1 macrophages. The identification of these genes will most likely provide clues for future detailed analysis by which TG impacts cholesterol homeostasis in macrophages.

Recent studies have shown that $\mathrm{ABC}$ transporters have important roles in efflux of endogenous cholesterols (Tarr and Edwards, 2008; Moore and Tabas, 2011). For example, combined deficiency of ABCA1 and ABCG1 resulted in a $60 \%$ decrease in macrophage cholesterol efflux in mice (Yvan-Charvet et al., 2007; Tall et al., 2008). In the current study, TG up-regulates ABCG1. It may suggest that cholesterol efflux is enhanced in response to TG. Also, it may be possible that TG may increase uptake of cholesterol resulting in up-regulation of ABCG1 to transport excess cholesterol outside of the cells for protection of the cells. Several proteins have been described in the literature that regulates ABCG1. One such protein is the liver $\mathrm{X}$ receptor (LXR). LXRs are transcription factors of the nuclear receptor superfamily and functions as a whole body cholesterol sensor (Calkin and Tontonoz, 2010). The ABC transportermediated efflux of cholesterol is regulated by LXRs and the bile acid receptor (FXR), as heterodimers with retinoid X receptors (RXRs) (Repa et al., 2000; Dean et al., 2001). Endogenous ligands for LXR are likely to be intermediates or end products of cholesterol metabolites such as hydroxycholesterol and epoxycholesterol. It was shown that LXRinduced expression of ABCA1 and ABCG1 in macrophages is essential for the stimulation of cholesterol efflux (Huwait et al., 2011; Moore and Tabas, 2011). Also other researchers have reported LXR agonists activate several components of the JNK pathway, such as SEK1, JNK and c-Jun. In addition, dominant negative mutation of JNK pathway inhibited the LXR agonist-induced up-regulation of $\mathrm{ABC}$ transporter in murine macrophages (Huwait et al., 2011). Paradoxically, Nagelin et al. showed that activation of ABCG1 is down-regulated by JNK pathway and p38 MAPK pathway (Nagelin et al., 2009). These results suggest that TG could possibly induce expression of ABCG1 via LXRs and JNK pathway. As for MEK1, studies have shown that the MEK1 pathway influences LXR-inducible ABC transporter expression in macrophages (Mulay et al., 2013). Similarly, our data also shows that TG negatively regulates
ABCG1 expression via MEK1 pathway. Overexpression of ABCA1 and ABCG1 protected macrophages against oxidative stress-induced cell death (Yvan-Charvet et al., 2010). In previous studies, we found that THP-1 macrophages treated with TG $(1 \mathrm{mg} / \mathrm{mL})$ for $24 \mathrm{~h}$ induced cell death in $30 \%$ of the cells (Son et al., 2013). It is possible that the induction of ABCG1 in TG-treated THP-1 cells may be result of a survival mechanism employed by the cells to prevent cell death.

In addition to SR-B1, macrophage scavenger receptor-1 (MSR1), MARCO and CD36 (also known as SCARB3) can all mediate the endocytosis of cholesterol by binding to modified low density lipoproteins (modified-LDLs). Although we found that SR-B1 expression was unaffected by TG treatment, it is possible that MARCO and CD36 may be affected. These possibilities will be evaluated in future experiments. In conclusion, our data showed that increase of exogenous TG induces up-regulation of ABCG1 expression through JNK and p38 MAPK pathway. These alterations maybe affects the cholesterol uptake/efflux. Although mRNA expression studies do not fully explain molecular mechanism of lipid accumulation, our data may provide further insights into the role of TG on macrophages during formation of foam cells.

\section{REFERENCES}

Aronis A, Madar Z, Tirosh O. Mechanism underlying oxidative stress-mediated lipotoxicity: Exposure of J774.2 macrophages to triacylglycerols facilitates mitochondrial reactive oxygen species production and cellular necrosis. Free Radical Bio Med. 2005. 38: 1221-1230.

Calkin AC, Tontonoz P. Liver X receptor signaling pathways and atherosclerosis. Arterioscler Thromb Vasc Biol. 2010. 30: 1513 -1518 .

Daniels TF, Killinger KM, Michal JJ, Wright RW, Jiang Z. Lipoproteins, cholesterol homeostasis and cardiac health. Int J Biol Sci. 2009. 5: 474-488.

Dean M, Rzhetsky A, Allikmets R. The human ATP-binding cassette (ABC) transporter superfamily. Genome Res. 2001. 11: 1156-1166.

Feingold KR, Shigenaga JK, Kazemi MR, McDonald CM, Patzek SM, Cross AS, Moser A, Grunfeld C. Mechanisms of trigly- 
ceride accumulation in activated macrophages. J Leukoc Biol. 2012. 92: 829-839.

Huwait EA, Greenow KR, Singh NN, Ramji DP. A novel role for c-Jun N-terminal kinase and phosphoinositide 3-kinase in the liver $\mathrm{X}$ receptor-mediated induction of macrophage gene expression. Cell Signal. 2011. 23: 542-549.

Kastelein JP, Steeg WA, Holme I, Gaffney M, Cater NB, Barter P, Deedwania P, Olsson AG, Boekholdt SM, Demicco DA, Szarek M, LaRosa JC, Pedersen TR, Grundy SM. Lipids, apolipoproteins, and their ratios in relation to cardiovascular events with statin treatment. Circulation. 2008. 117: 3002 -3009 .

Kennedy MA, Barrera GC, Nakamura K, Baldan A, Tarr P, Fishbein MC, Frank J, Francone OL, Edwards PA. ABCG1 has a critical role in mediating cholesterol efflux to HDL and preventing cellular lipid accumulation. Cell Metab. 2005. 1: 121-131.

Khera AV, Cuchel M, de la Llera-Moya M, Rodrigues A, Burke MF, Jafri K, French BC, Philips JA, Mucksavage ML, Wilensky RL, Mohler ER, Rothblat GH, Rader DJ. Cholesterol efflux capacity, high-density lipoprotein function, and atherosclerosis. N Engl J Med. 2011. 364: 127-135.

Kunjathoor VV, Febbraio M, Podrez EA, Moore KJ, Andersson L, Koehn S, Rhee JS, Silverstein R, Hoff HF, Freeman MW. Scavenger receptors class A-I/II and CD36 are the principal receptors responsible for the uptake of modified low density lipoprotein leading to lipid loading in macrophages. J Biol Chem. 2002. 277: 49982-49988.

Lind L. Circulating markers of inflammation and atherosclerosis. Atherosclerosis. 2003. 169: 203-214.

Moore KJ, Tabas I. Macrophages in the pathogenesis of atherosclerosis. Cell. 2011. 145: 341-355.

Mulay V, Wood P, Manetsch M, Darabi M, Cairns R, Hoque M, Chan KC, Reverter M, Alvarez-Guaita A, Rye KA, Rentero C, Heeren J, Enrich C, Grewal T. Inhibition of mitogenactivated protein kinase Erk1/2 promotes protein degradation of ATP binding cassette transporters A1 and G1 in CHO and HuH7 cells. PLoS One. 2013. 8: e62667.

Nagelin MH, Srinivasan S, Nadler JL, Hedrick CC. Murine 12/ 15-lipoxygenase regulates ATP-binding cassette transporter G1 protein degradation through p38- and JNK2-dependent pathways. J Biol Chem. 2009. 284: 31303-31314.

Palmer AM, Murphy N, Graham A. Triglyceride-rich lipoproteins inhibit cholesterol efflux to apolipoprotein (apo) A1 from human macrophage foam cells. Atherosclerosis. 2004. 173: 27-38.

Repa JJ, Liang G, Ou J, Bashmakov Y, Lobaccaro JM, Shimomura I, Shan B, Brown MS, Goldstein JL, Mangelsdorf DJ. Regulation of mouse sterol regulatory element-binding protein-1c gene (SREBP-1c) by oxysterol receptors, LXR $\alpha$ and LXR $\beta$. Genes Dev. 2000. 14: 2819-2830.

Son SJ, Rhee K-J, Lim J, Kim TU, Kim TJ, Kim YS. Triglycerideinduced macrophage cell death is triggered by caspase-1. Biol Pharm Bull. 2013. 36: 108-113.

Tall AR, Yvan-Charvet L, Terasaka N, Pagler T, Wang N. HDL, $\mathrm{ABC}$ transporters, and cholesterol efflux: implications for the treatment of atherosclerosis. Cell Metab. 2008. 7: 365-375.

Tarr PT, Edwards PA. ABCG1 and ABCG4 are coexpressed in neurons and astrocytes of the CNS and regulate cholesterol homeostasis through SREBP-2. J Lipid Res. 2008. 49: 169 -182 .

Wang N, Silver DL, Thiele C, Tall AR. ABCA1 functions as a cholesterol efflux regulatory protein. J Biol Chem. 2001. 276: 23742-23747.

Yancey PG, Bortnick AE, Kellner-Weibel G, de la Llera-Moya M, Philips MC, Rothblat GH. Importance of different pathways of cellular cholesterol efflux. Arterioscler Thromb Vasc Biol. 2003. 23: 712-719.

Yoon HS, Ju JH, Lee JE, Park HJ, Lee JM, Shin HK, Holzapfel W, Park KY, Doa MS. The probiotic Lactobacillus rhamnosus BFE5264 and Lactobacillus plantarum NR74 promote cholesterol efflux and suppress inflammation in THP-1 cells. J Sci Food Agric. 2012. 93: 781-787.

Yvan-Charvet L, Ranalletta M, Wang N, Han S, Terasaka N, Li R, Welch C, Tall AR. Combined deficiency of ABCA1 and ABCG1 promotes foam cell accumulation and accelerates atherosclerosis in mice. J Clin Invest. 2007. 117: 3900-3908.

Yvan-Charvet L, Pagler TA, Seimon TA, Thorp E, Welch CL, Witztum JL, Tabas I, Tall AR. ABCA1 and ABCG1 protect against oxidative stress-induced macrophage apoptosis during efferocytosis. Circ Res. 2010. 25: 1861-1869. 